\title{
Architectural Design Students' Explorations through Conceptual Diagrams
}

\author{
Fehmi Dogan \\ Assistant Professor of Architecture, \\ Izmir Institute of Technology, Izmir, Turkey
}

ABSTRACT Views of creativity highlight the importance of incubation or the significance of sketching as a means of seeing emergent properties. Both views put design students at a disadvantage. This study investigates the strengths and weaknesses of an alternative approach to design education, in which students were asked to develop a design idea through conceptual diagrams. This study investigates how conceptual diagrams might help architectural students to see the relationships between concepts and space and coordinate their dual development through conceptual diagrams. The study presents the development of the ideas of 13 second-year architectural students. Students' logbooks, together with their midterm and final review presentations, 
were studied to determine whether students drew

any conceptual diagrams, whether they were

instrumental in spatial organization, and how they

introduced changes during the design process.

The findings showed that this particular design

education approach helped students start the

design process and stay focused throughout the

design process.

KEYWORDS: design education, conceptual design, conceptual diagrams

\section{Introduction}

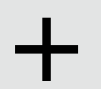

Architectural design situations often impose both conceptually and spatially challenging tasks and require a

coordinated exploration of the two. One of the difficulties for novice architectural students during the initial phase of design is the struggle to formulate an abstract design concept and relate this idea to spatial arrangements. Often students sketch with the hope of stumbling upon an idea or they establish a quick and superficial correspondence between an abstract idea and a spatial arrangement.

This study investigates the design processes of students in a second year level undergraduate architectural design course and inquires how concept generation and concept development might be facilitated through a series of related tasks assigned during the initial phase of design. It is conjectured that conceptual diagrams could potentially help students think about relationships between their abstract design concepts and architectural space and also conjectured that design exploration might be structured through the use of conceptual diagrams. In this study a conceptual diagram is defined as a visual/spatial configuration which is representative of the core of a design conceptualization and which highlights the structure of the design conceptualization through its spatial configuration. Studies of expert architects' design processes have shown that conceptual diagrams are representational tools that help these designers think about abstract ideas and spatial schemes simultaneously (Dogan, 2003; Dogan and Nersessian, 2010; Dogan and Zimring 2002). It is proposed that a structured approach using conceptual diagrams could provide a productive alternative to teaching methods in which students are encouraged to sketch constantly, with the hope of discovering unexpected emergent properties in these sketches. Sketches are shown to be useful in the creative process; however, an exclusive reliance on sketching for idea generation may put novice students at a disadvantage because they do not know what they are looking for in the sketches.

A pedagogical challenge of design education is to get students 
product. Schön $(1984,1987)$ characterized the design studio environment as a place in which thinking occurs through a dialectic conversation between the design instructor and the student, and through the medium of external representations such as sketches. Goldschmidt (1994) presents a case in which a student who is stuck at the beginning of the design process repeatedly scribbles his signature and thus stumbles upon an original idea that breaks the impasse. Accepting that students need to have constant coaching and dialectic reflection, as Schön suggests, and recognizing the possibility of serendipitous discovery through sketches, as reported by Goldschmidt, it is suggested here that students could also benefit from relatively more structured design education environments. This paper reports the results of a study in a second-year undergraduate design studio in which the process of constructing abstract design ideas and developing those ideas was relatively more structured through the assignment of distinct but related preliminary design tasks, using conceptual diagrams. These preliminary design tasks were as follows:

1. Formulate a generic design concept.

2. Represent the design concept graphically in the form of conceptual diagram(s).

3. Find precedents for the design concept and analyse how some aspects of the precedents might be transferred to the current design task.

4. Create variations on the design concept, in order to see how a generic design concept could be materialized in different, but related, spatial schemes.

5. Manipulate the variations of the conceptual diagram(s), to facilitate conceptual change and generate breakthroughs in the design process.

This pedagogical approach comes from the belief that a conceptdriven design process will help students stay committed and motivated during the design process. An illustration of a concept-driven design process may be found in Louis I. Kahn's description of how design proceeds from 'form' to 'design' (Kahn, 1961). A critical reading of Kahn will show that the progression of his designs from concept to design was never linear and straightforward, as he suggests, yet, such a reading will also show that design concepts and their diagrammatic representations are significant in Kahn's design process (Dogan and Zimring, 2002). Here, it is proposed that conceptual diagrams, because they are intrinsically spatial representations, might help students relate abstract ideas to generic spatial schemes (Dogan, 2003). These diagrams, as external representations that are linked to mental models, are easily modified and such modifications might trigger corresponding changes in the designer's generic conceptualization of a solution. 
Dogan (2003) proposes that expert designers have a structured mental model with conceptual as well as spatial components, in relation to Johnson-Laird's insight concerning the relation between diagrams and mental models (Johnson-Laird, 2002), Greeno's proposal that manipulating physical models can provoke corresponding changes in mental models (Greeno, 1989), and Nersessian's argument that conceptual innovation often involves simulating a mental model (Nersessian, 2008). Johnson-Laird (2002) characterized diagrammatic representations in terms of a correspondence to a target domain, wherein the structure of the two domains remains the same. According to Johnson-Laird the correspondence between diagrams and their target domains is in structure and not in any other feature. Johnson-Laird also claims that mental models maintain structural correspondences to their represented domains but notes that mental models are internal representations whereas diagrams are external representations that correspond to a mental model. As such, diagrams are what Greeno (1989) considered to be physical models of structured mental representations. The significance of a physical model with correspondence to a mental model is that the physical model can literally be simulated and inspected for inferences. Thus, manipulation of conceptual diagrams could trigger mental simulations that might lead to innovation.

\section{Initial Phases of Design}

Some expert architects, such as Le Corbusier and Frank Lloyd Wright, have described the initial phase as a period of incubation at the end of which the design idea emerges suddenly and automatically. These architects, in their own accounts of their design processes and in their writings on design, suggest that they first acquire a full understanding of the design situation as if collecting information in a black box and then start translating their ideas onto paper (Le Corbusier et al. 1981; Pfeiffer and Wright, 1990).

The accounts of Wright and Le Corbusier seem to be in agreement with a particular characterization of creativity according to which the creative moment is preceded by a period of incubation and followed by a period of implementation (Boden, 1991). Even though incubation may have some positive role in creativity, we know that the accuracy of Wright's and Le Corbusier's accounts of their design processes in the early stages of design is questionable (see Hoffmann, 1978; Le Corbusier et al, 1981). Other studies of expert architects, such as Kahn, Libeskind and Stirling, have shown that the initial stages of design may require intense thinking and working (Dogan, 2003). Moreover, a black-box view of design would suggest that design teaching is an almost impossible task.

Some studies that have focused on designers' behaviours in the early stages of design cast doubt on expert architects' accounts. These studies indicate the significance of the early stages of design, during which designers construct a better understanding 
of the design situation. Schön $(1984,1988)$ from a constructivist perspective, highlighted the importance of what he calls 'problem framing' in initial phases of design. Goel and Pirolli (1992) suggest that problem-solving in design starts only after the problemstructuring phase. Some studies (Lawson, 1979; Lloyd and Scott, 1994) provide evidence for some of the expert designers' accounts, suggesting that designers begin problem-solving by focusing on solutions first (Lawson, 1979) or that designers with experience in a particular design task begin with a solution (Lloyd and Scott,1994). Co-evolutionary views of design (Dorst and Cross, 2001; Gross et al, 1987; Maher and Tang, 2003; Suwa et al, 2000) suggest a process in which problem solution and problem-structuring evolve simultaneously.

In some of the studies referred to above, it is suggested that designers are involved in an intense effort of sketching early in the design process. In these studies sketching is proposed as the main medium through which designers see emergent properties and formulate them into unexpected ideas. Different from the black-box view of design, these studies highlight the designers' intense sketching efforts in the early phases of design and characterize these efforts as an almost-blind search in a vast terrain. This suggests that through sketching some students might stumble upon useful ideas but others might not.

The literature on sketching is replete with cases in which sketching is proposed as the primary medium through which students can explore their ideas. Goldschmidt (1991, 1994, 1997), Schön (1987, 1992), and Schön's colleagues (Schön and Wiggins 1992) have highlighted the importance of sketches in the dialectic interaction between thoughts and marks on paper. Common to Goldschmidt and Schön is the idea that design evolves incrementally through local moves towards a Gestalt shift. For both incremental changes and sudden changes, discovery appears to be accompanied by a sense of surprise. The crucial cognitive task in this process is recognition of the consequences. To appreciate the value of the unintended consequences one has to be able to continuously reinterpret the marks on the paper. This is relatively more straightforward for expert designers compared to students because of differences in the structure of their mental representations, as suggested by Chi and her colleagues (1981). Chi et al found that, when solving physics problems, expert physics problem-solvers invoke an appropriate schema which is a principle-oriented knowledge structure, whereas novices do not have such structured knowledge representations. Here it is suggested that expert designers construct mental representations of design situations that are enriched with precedents, heuristics and domain knowledge, whereas students' mental representations do not include some or any of these. Thus it is suggested here that if students rely on sketching exclusively it puts them at a disadvantage. 
This study inquires whether students' creative processes could be fostered through the use of less ambiguous types of representation, such as conceptual diagrams. Such diagrams might be coupled with the use of sketches to facilitate unexpected discoveries. This more structured exploration might improve student/teacher dialogue throughout the design process, improve design task outcomes for novice designers and provide evidence that design is not only a serendipitous process of discovery.

Research in the area of diagrammatic reasoning has shown that diagrammatic representations, different from other kinds of representations, directly represent the structure of their target domain (Shimojima, 2001; Stenning and Lemon, 2001) and thus simplify the complexity of conceptual domains (Bauer and Johnson-Laird, 1993; Gattis and Holyoak, 1996; Gobert and Clement, 1999).

In design studies literature some studies on diagrams highlight the benefits of diagrams in the design process. According to Alexander (1964), diagrams are helpful because they structure the design problem into sub-components. Clayton's (2000) and Oxman's (1997) works suggest that diagrams help analyse the structure and characteristics of existing buildings. Do's work (Do and Gross 2001) shows that diagrams are extensively used by designers. Ervin (1990) suggests that diagrams are intermediary representations between initial concepts and final detailed drawings. The results of these studies, together with the diagrammatic reasoning literature, suggest that diagrams could be useful for students as well.

\section{Study}

The study presents the evolution of the design ideas of 13 secondyear architectural design students given an architectural design task, based on their design logbooks and documentation of the state of their works during two midterm reviews and one final review by instructors. In this course students were asked to design an oral history centre and were given two months to complete the project. The site of the project was located between the remains of a Roman road and a city park in Izmir, Turkey. The design task emphasized the public role of the building and introduced a particular design theme, integration, to guide students' thinking about the design task.

Student logbooks, together with midterm and final review presentations, were studied to determine if students drew conceptual diagrams and used them in spatial organization, and how they introduced changes during their design process. The logbooks provided the students' perspectives of their own design process, and the studio instructors documented the students' midterm and final presentations, which included drawings and models. The logbooks provided a body of material of what the students were thinking about design processes, while the presentation materials illustrated the level that had been achieved at particular moments in the process. Drawings and notes from the logbooks and from the reviews were 
analysed to identify the conceptual underpinnings of the students' design ideas and to determine whether these ideas were expressed in diagrammatic representations. Subsequently, schematic variations were analysed to find out how students proceeded from generic ideas to specific design schemes. Finally, the logbooks and drawings were examined to determine whether there had been any significant conceptual shifts during the design process and how these shifts had been facilitated.

\section{Students' Design Processes}

Five main points of the students' design processes were investigated: (1) how the students generated a design idea; (2) whether and how these ideas were expressed diagrammatically; (3) what functions these diagrams had in the design process; (4) how students converted their design ideas into specific spatial schemes; and (5) whether and how students shifted their conceptual understanding, i.e. made breakthroughs.

\section{How the students generated design ideas}

In developing a design concept students were encouraged to think about three main issues: the design problem definition, the architectural programme and the site. The design problem definition highlighted the role of documenting and sharing cultural history and introduced the theme of integration, both among people and between the building and the site. The architectural programme emphasized the social and research activities which were to be housed within the centre. Finally, the site was on a slope between an urban park and archaeological remains, with a commanding view of the Izmir Bay, and surrounded by a diversity of functions and people. Proposed concepts were evaluated based on considerations of these three issues. Specific tasks used to facilitate concept generation were site analysis, programme analysis and problem reinterpretation. Through site analysis students became familiar with the social, physical, functional and natural features of the site. Through programme analysis students were able to identify the important activities and functions within the building. To help them reinterpret the problem definition students were asked to think about 'what the building wants to be' (as expressed by Louis I. Kahn) and for whom this building was going to be built. The initial concepts students formulated were discussed with studio teachers, both individually and in groups.

Upon examining students' first ideas it became evident that students had often used analogies to construct a series of related concepts. In this initial stage each student was encouraged to think as openly as possible, with the idea that personally meaningful concepts which express an individual response and individual significance assigned to particular features of the design situation might 


\section{Figure 1}

Student A's conceptual diagrams showing generic instances of 'path'. thus become the basis for devising a spatial configuration. Students were then encouraged to provide spatial reciprocals of their own analogical inferences, via diagrammatic expressions.

Of all of the students, nine offered analogies to begin the design. Students' analogies ranged from familiar building types or structures such as home to abstract entities such as history. The building or structure types of analogies included path, bridge, city, village teahouse, street market and home as the source domains. More abstract analogies included witness to history, history and an interactive relationship between memory and history. Other students offered non-analogical idea conceptions, for example, a focus on specific site issues, such as shifting views going from the top of the site (Roman road) to the bottom (park). Two students' design concepts related to the occupants' spatial experiences. One suggested that the building should foster unintended experiences for its occupants while the other focused on light and the expressive use of light in the building.

Most of the students' analogies were within-domain analogies, i.e. they were limited to the architectural domain. Fewer students established analogical relationships with remote domains. JohnsonLaird (1989) claimed that profound analogies are harder to establish, which could explain why more students used within-domain analogies. Contrary to Johnson-Laird and to the findings of this study, Casakin (2004) found no difference between expert designers and novice designers in accessing remote domain analogies. One difference is that in Casakin's study the source domain examples, for both within and between domain analogies, were available to students before the design process; therefore, novice students were prompted to pick remote source examples. In this study the source domains were established by the students themselves, and more

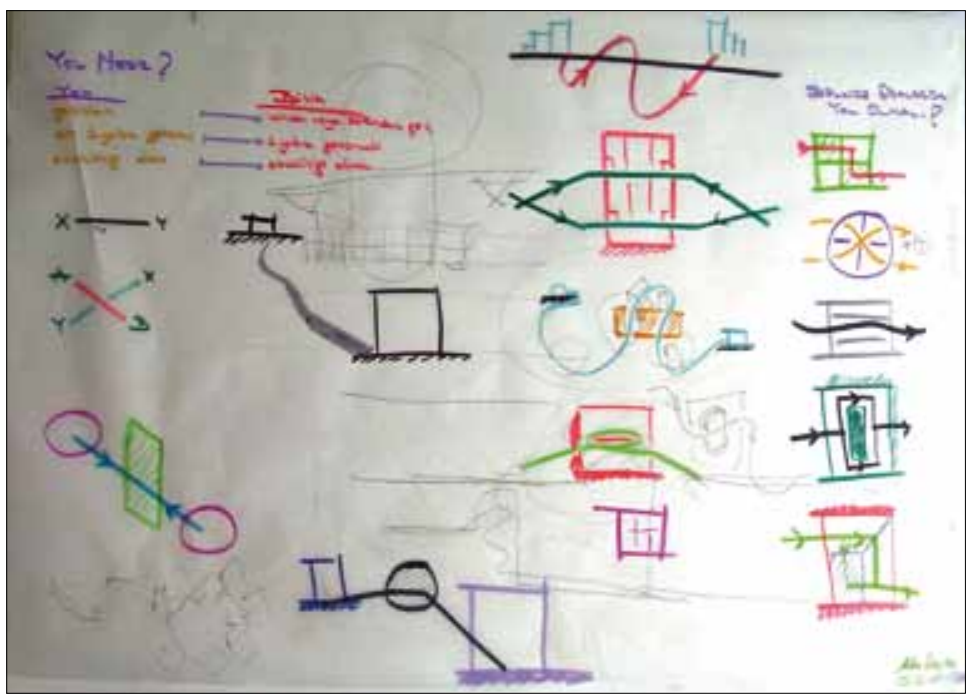




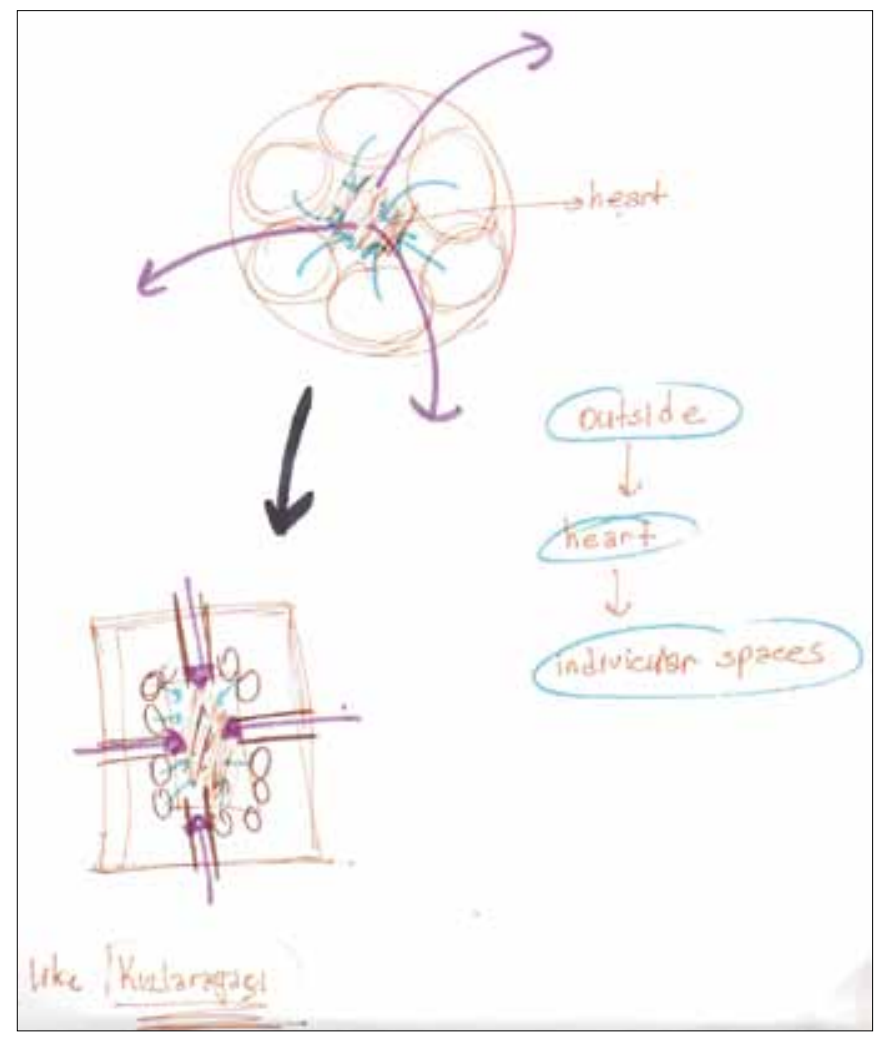

students preferred architectural examples from which they could transfer spatial features to their design situation.

One of the primary strengths of metaphors and analogies is the ability to project the attributes of a familiar situation to an unfamiliar situation (Gentner, 1983; Lakoff, 1987). For students, design situations often present unfamiliar territories, which may explain why students in this study primarily used analogies in idea generation. Casakin (2006) found that for design students the use of metaphors is easier in the early stages of design, also called the conceptual phase, whereas in the later stages it is more difficult. Here it is observed that often their own analogies offered students a group of related ideas and spatial characteristics about a particular situation. For instance, the village teahouse analogy brings with it the concepts of publicness and social contact, together with a certain sense of scale, and a feeling of cosiness and togetherness. During individual and group discussions analogies provided by the students were elaborated to further study their conceptual and spatial implications.

\section{Diagrammatic expressions of students' initial design concepts}

Following the formulation of design concepts students were required to represent their concepts diagrammatically. The main purpose

\section{Figure 2}

Student B's initial conceptual diagrams. 


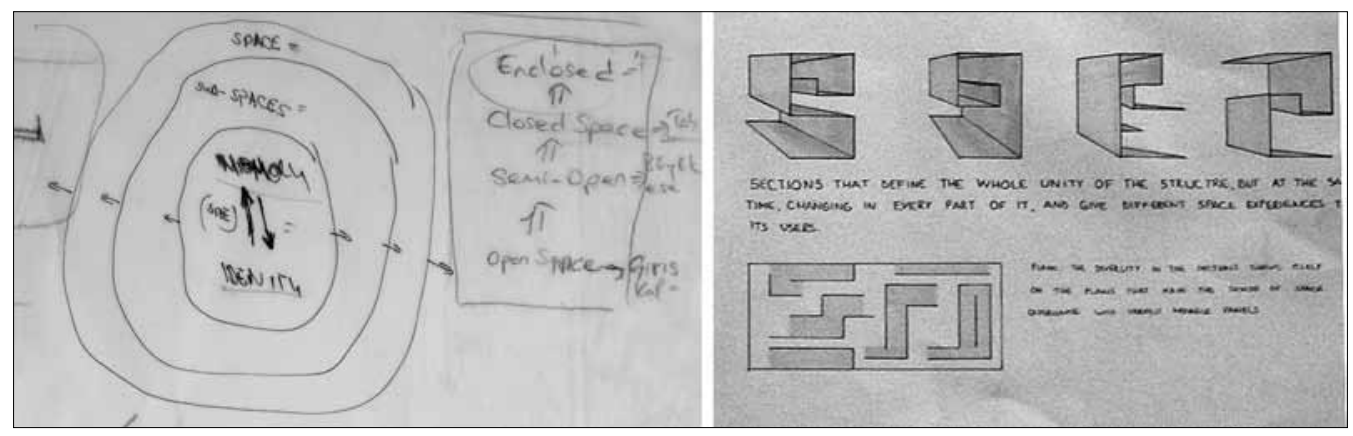

Figure 3

Student C's initial conceptual and section diagrams.

\section{Figure 4}

Student D's initial conceptual diagrams. was for students to translate their abstract ideas into generic spatial configurations and thus facilitate the transition from idea generation to spatial thinking. Some of these diagrams were generic representations of design ideas. There were, however, other types of diagrams students drew repeatedly, such as bubble diagrams exploring functional and adjacency relationships, circulation diagrams, zoning diagrams and section diagrams. Most often, these diagrams suggested a spatial organization, because diagrams themselves are spatial. However, less often they also referred to an abstract idea.

Here, a few examples of students' diagrams from the many considered are studied in detail. The diagrams that are shown here

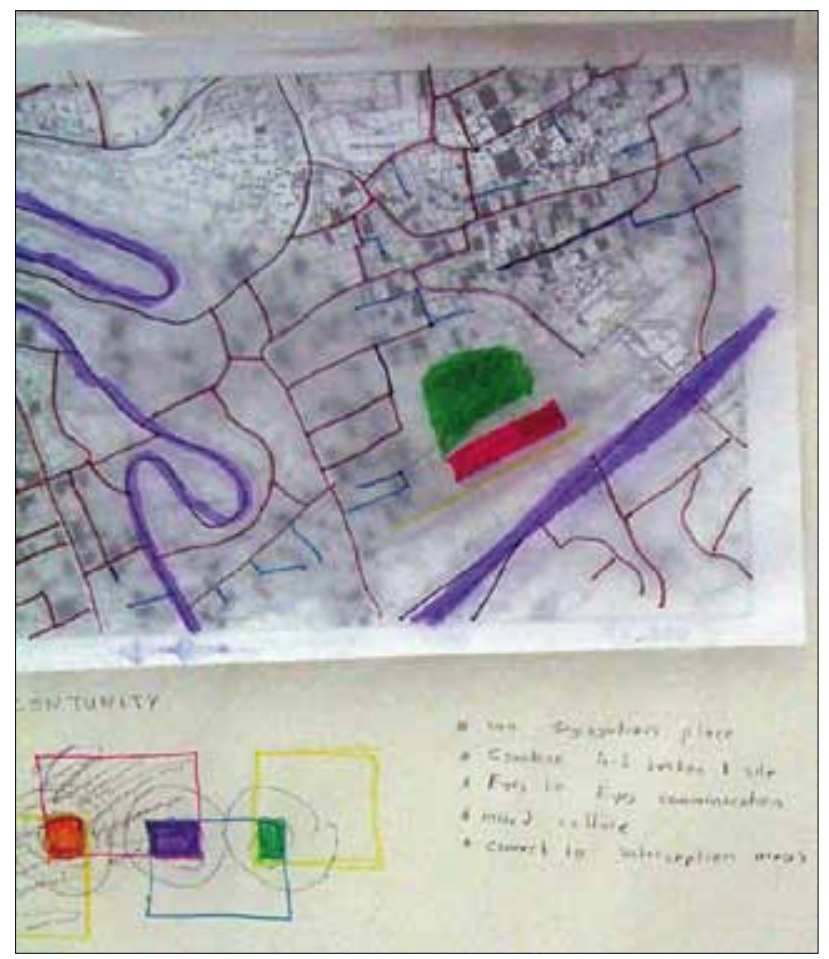




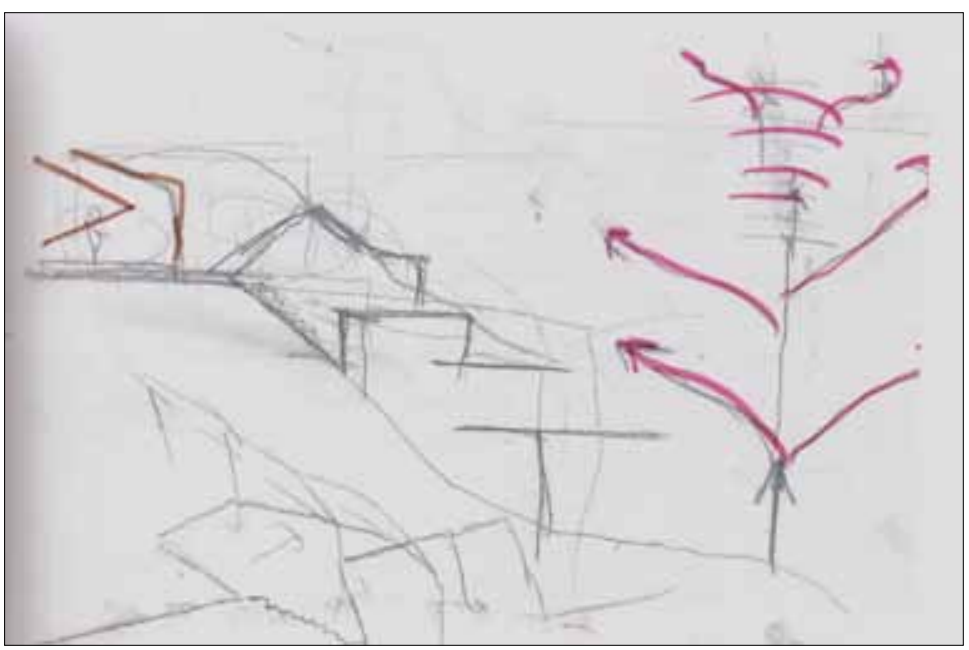

Figure 5

Student E's initial

conceptual diagrams.

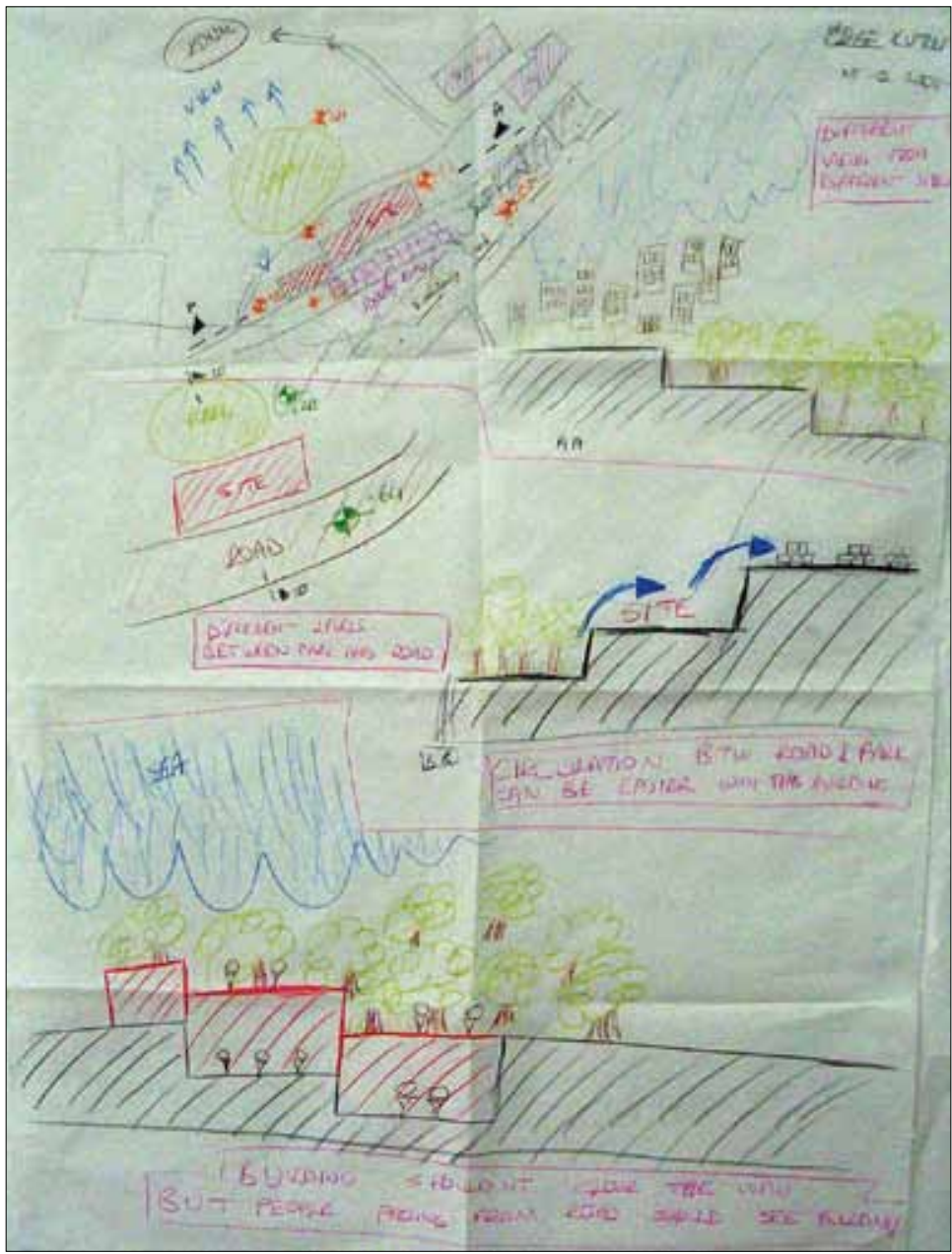

Figure 6

Student F's initial

conceptual diagrams. 


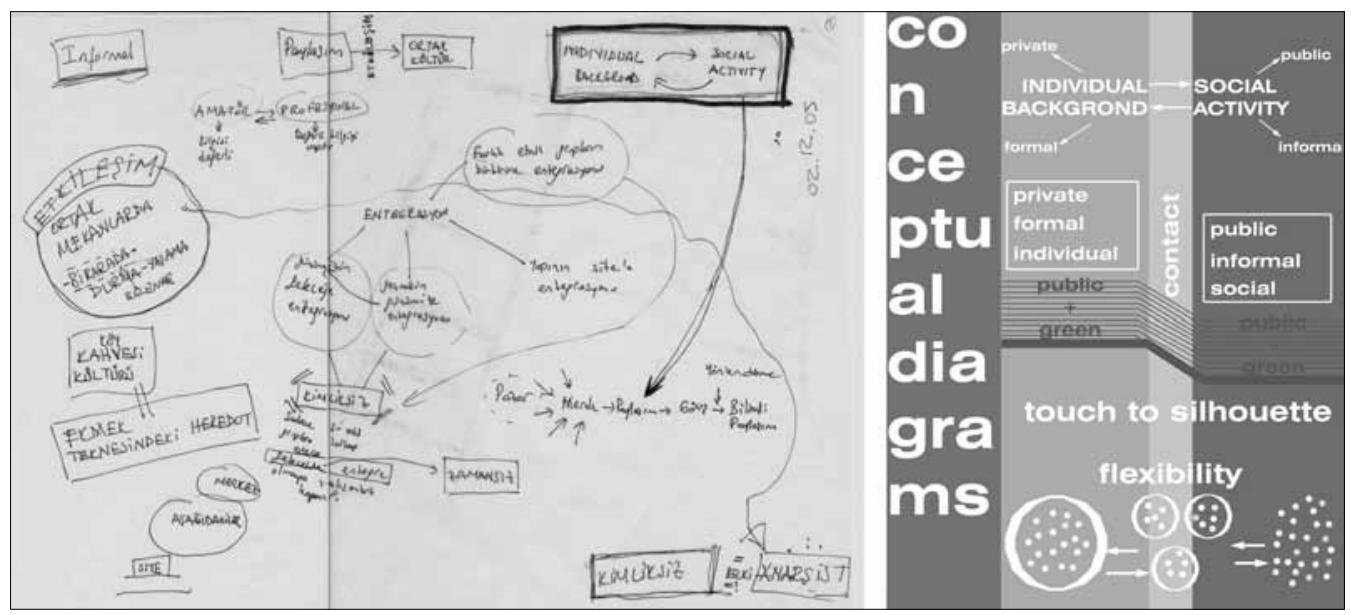

Figure 7

Student G's initial conceptual diagrams and final ones from his portfolio. clearly demonstrate a design commitment in the design process. Student A started with a path analogy and drew generic representations of his source domain, the path. He drew several instances of path, including paths going through different generic buildings (Figure 1). In these diagrams this student conceptualized history as a journey, thought about the trajectory of a visitor through the site and the building, and thought about how this trajectory would change according to the shape of the path. The analogy evoked both an event, i.e. a journey, and a spatial scheme, i.e. a trajectory from $A$ to $B$, and both are represented by his diagrams. For Student $A$ the diagram aligns his conceptualization of the design problem with generic spatial schemes.

Other students formulated new ideas through diagrammatic explorations. Student B drew a series of diagrams with which she conceptualized relationships between the building and its surroundings. In one of the diagrams the student was concerned with a close connection between the building and its surroundings (Figure 2). In a subsequent diagram she introduced relationships between the surroundings and individual spaces of the architectural programme, originating in a space she called heart, denoting the centre of the building. The heart of the building had a direct link with the outside and the individual programme components.

Student C was concerned with the analogical relationship between memory and personal identity. He drew a diagram in the form of concentric, permeable rings which he related to the layers of personal memory which form an individual (Figure 3). He also sketched a spatial analogy that showed the layers of memory as concentric spaces which were transparent. In the spatial scheme he introduced a succession of spaces, graduating from open, to semi-open, to closed. In his subsequent diagrams, Student $\mathrm{C}$ translated his idea of concentric circles into section diagrams through which he explored 
how spaces of the architectural programme could wrap and enclose each other. For this student, use of diagrams facilitated the translation of a seemingly abstract and evocative idea into a corresponding spatial scheme.

Student D diagrammatically analysed the urban fabric of the surrounding neighbourhoods (Figure 4) and identified segregation, dead-ends, lack of commercial life and isolation from the rest of the city as problems in the area. In response he imagined a series of flowing spaces inside the building and continuity outside the building by connecting the park and the Roman road.

A small group of students derived design ideas from the topographical condition and other environmental factors. Student E thought about layering the building along the slope and branching the building circulation, while creating unintended experiences for its occupants (Figure 5). Student F focused on changing view as one descends from the Roman road to the city park and aimed to use the building to provide an easy transition from the road to the park (Figure 6). Student E and Student F both used diagrams as analytical tools to help establish the relationship between the building and its site.

Student $G$ explored relationships between different ideas. He used diagrams to map relationships between different but related concepts that were meaningful to him (Figure 7). One particular diagram became instrumental in the design process. This diagram showed a bidirectional relationship between society and the individual. This duality suggested a spatial division which the student employed to divide the building programme into two chunks and place them in separate zones.

In those instances where students' initial ideas relate directly to a spatial source domain, such as path (Student A), the translation to diagrams was more straightforward. In instances where the initial idea had no direct spatial connotations, such as memory and identity (Student C), social and individual (Student G) or segregation (Student D), diagramming functioned in two ways. First, it helped students formulate generic spatial schemes. Second, it helped students explore, study and think about complex conceptual domains. Gattis and Holyoak (1996) showed that complex non-spatial problems could be simplified through spatial representations. It is possible that the students' diagrams of this study worked in a similar way.

\section{What were functions of these diagrammatic expressions?}

The reasons for asking students to draw conceptual diagrams are many. First, through the use of mediating representations such as diagrams students may acquire an understanding of how abstract concepts can be used in the design process. Students may also begin to understand how such concepts can then be translated into spatial schemes. Second, students' diagrams often serve as 


\section{Figure 8}

Student A's diagrams studying a different generic scheme.

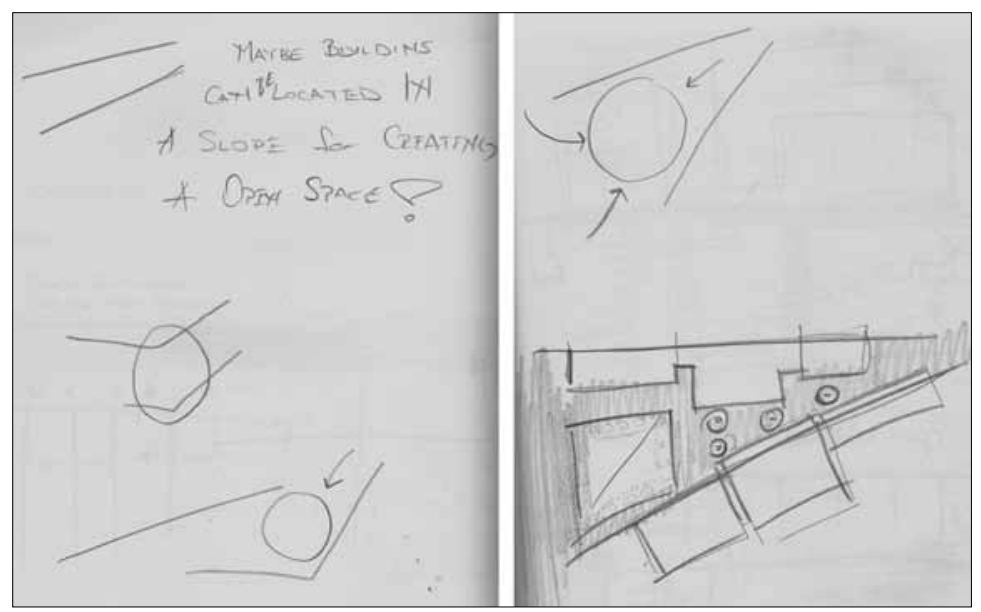

documents of decisions in time, of commitments to design ideas. Third, manipulating diagrams at a generic level may lead to significant conceptual and spatial breakthroughs for the novice designers.

Most students realized that their diagrams were generic abstractions and not simplified spatial schemes, but failed to follow the spatial implications of those generic schemes through different variations and fully explore the spatial alternatives suggested by their initial generic abstractions. Often students did develop their initial generic schemes further in response to requirements for more detailed development of the design. In these instances the generic diagrams helped students stay committed to a design idea without getting lost in the design process. Coyne and his colleagues (1994) proposed that metaphors privilege certain aspects of a design situation and through this the designer establishes 'a focus of concern'. Similarly diagrams bring a focus to the design exploration.

One difficulty that the students of this study experienced relating to formulating a clear design concept was the early fixation to an idea before evaluating and comparing other ideas. This would be particularly true if the initial conceptual phase of design is rushed, without giving students enough time and opportunity to think about the different dimensions of the design situation. To avoid this problem, students might be asked to re-evaluate their initial concepts whenever new design issues emerge or are introduced into the design space. As a design proceeds from more general considerations to more specific ones, the design concept should be re-evaluated and, if necessary, be modified according to emerging requirements of the design problem.

During this study, when problems arose during the design process students usually went back to their original conceptual diagrams. Some actually modified the original diagrams to break an impasse in the design situation. One such example is illustrated by the design 


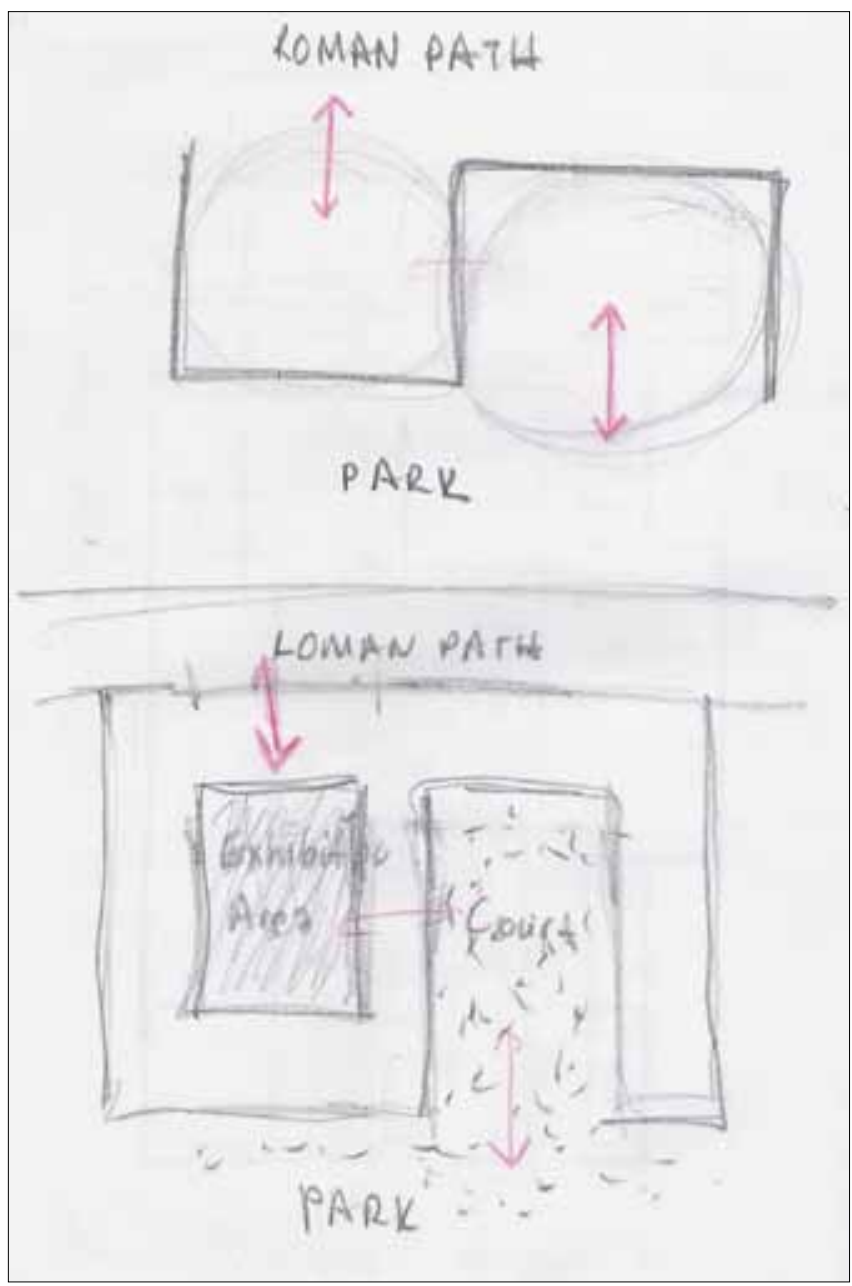

process of Student A, who started with the idea of a path. Student A quickly translated this idea into two parallel bars with a path between them. Spatially, the scheme did not carry the richness and variety of his generic diagrams. In addition, the student did not consider the topography when adopting his scheme into its setting. He realized that the parallel bars could follow the topography and curve accordingly. In a series of diagrams he studied how the two bars could have different angles and, in doing so, discovered that if he changed the angles between the bars he could create both an open public space and a path through the building (Figure 8). The case of Student A is a good illustration of how an initial concept may be revised in response to a new design parameter, in this case topography. Furthermore, it is one of the few examples in this study in which manipulation of the diagram introduced a new spatial element to the scheme, in this case the addition of an open public space along the path.

\section{Figure 9}

Student B's manipulation of her initial diagrams. 
Students often had difficulties relating their conceptual diagrams to spatial schemes. They were instructed to find precedents and sometimes were given specific examples to study and from which to derive the spatial organizing scheme through diagrammatic analysis. As Coyne and his colleagues (1994) suggest, in this study, looking at precedents helped students 'see this situation as another situation' (1994: 117) and facilitate the transfer of successful features of an example to a design situation in hand. One student studied the free plan arrangement in the work of Mies van der Rohe; another studied traditional Japanese houses; another studied Mies' Crown Hall and Richard Rogers and Renzo Piano's Pompidou Centre. In each instance students were asked to diagrammatically analyse and abstract the precedent. The following is a more detailed account of one student's use of precedent and her diagrammatic analyses.

Student B (heart scheme) looked at a local historical building, an Ottoman inn, which she abstracted diagrammatically to try to understand its circulation system. The study of this particular building helped her see how one can enter the centre of a building directly. Then she explored a plan scheme with a meeting room and exhibition space in the centre, both of which had direct access to the outside. In a subsequent diagram she drew the same scheme with the central space slightly shifted. In a new generic scheme two central spaces were placed next to each other and slightly shifted. One central space was the meeting room, which she opened directly onto the Roman road; the second central space was the exhibition space, which she opened directly to the park. She created a direct passage between the two central spaces. On the advice of her instructor she then replaced the meeting room with an outdoor courtyard and, in doing so, discovered a new spatial scheme in the shape of a tilde (Figure 9). In this particular instance we see how a student's project evolved from a generic scheme to a relatively more detailed scheme and back to a new generic scheme. Diagrammatic manipulations helped this student configure a richer spatial solution.

\section{Benefits of working with conceptual diagrams: From concept to space}

The first benefit of working with conceptual diagrams relates to the way students conduct their design explorations. Each student in the study formulated a design idea and proceeded with it through subsequent phases of design. Students had no problem starting the design process even when they had to change their initial idea. This is significant with regards to some different characterizations of design, each of which highlights difficulties for students. Goel and Pirolli's view (1992) suggests that before beginning a design designers should be equipped with the appropriate analytical skills, which students often do not have in the initial years of their education. Lawson's view (1979) suggests that students should start with a solution, however, they do not have sufficient knowledge of relevant precedents. 
A second benefit of working with conceptual diagrams was that students acquired more understanding of the process of design. As long as their initial ideas met the requirements of the design situation students were encouraged to follow them during their design explorations and consider variations of the initial concept rather than seek new ideas. Students were encouraged to modify their conceptual diagrams in response to emerging requirements. Such modifications in the conceptual diagrams might have provided opportunities for radical shifts; however, none of the students went that far in their explorations. The students who benefited most from modifying their diagrams were those who understood that their ideas were generic. These students focused on the generic spatial schemes suggested in their diagrams and further studied these schemes in precedents and through different variations. Often, looking at precedents with an analytical eye empowered students to see themselves as architects who could get inspiration from others. Furthermore, it convinced them that their schemes were generic and could be applied to different problems; therefore, they could explore the same idea in several variations. Using diagrams to look at precedents in this way encouraged students think about spatial relationships rather than superficial similarities. This coupling of the use of conceptual diagrams and precedents is worth highlighting because, as suggested by Oxman and Oxman (1992), model-based reasoning and case-based reasoning are two distinct cognitive strategies in design. In model-based reasoning design starts with generic schemes and evolves through what Oxman and Oxman call refinement. In casebased reasoning design starts with specific examples and evolves through adaptation. In this study students were encouraged to use both strategies. This coordinated use of both strategies highlighted both the generalities of conceptual diagrams and the specifics of precedents.

A third benefit of working with conceptual diagrams was that students came to understand that the formulation of abstract ideas and the development of generic spatial schemes need to be coordinated. Teaching this was attempted through requesting construction of conceptual diagrams to represent both abstract ideas and generic spatial schemes.

Based on this study it is conjectured that constructing physical representations such as conceptual diagrams could help novices build structured mental representations in the form of mental models. Furthermore, manipulating such representations might evoke a corresponding simulation of the designer's mental model to facilitate innovation. This assumes that the designer's mental model is coupled with external representations and that the designer is aware of this coupling.

Above all, an explicit attempt on the part of the students at formulating a design concept and expressing it in diagrammatic representations enhanced communication between instructors and 
students. Such generic representations are easily memorable and graspable by eye, and thus instructors may more easily follow students' progress.

\section{Difficulties for students: Conceptual shifts}

Study of students' logbook drawings showed that some students (six out of 13) acquired an understanding that conceptual diagrams may represent a design idea. Some students (seven), however, drew bubble diagrams or zoning diagrams, even though they were instructed in conceptual diagrams. Among the six students who drew conceptual diagrams, five seemed to understand that conceptual diagrams may represent both abstract ideas and ideas about spatial planning, and only two seemed to understand that conceptual diagrams are generic. These two students were able to consider variations of the same generic idea through different spatial schemes. In contrast to most students' use of conceptual diagrams in this study, expert designers such as Louis Kahn show us how the same conceptual diagram can help generate different spatial variations (Dogan and Zimring, 2002) and how changes in the conceptual diagram may bring simultaneous, coordinated changes in the conceptual and spatial domain (Dogan and Nersessian, 2010).

For the students in this study it seemed to be even harder to manipulate their generic diagrammatic representations in order to look for emergent ideas that might foster conceptual changes. Kahn's work, for instance, suggests a rather different discovery process, in which a designer not only sketches to see and inspect emergent properties but manipulates relatively less ambiguous components of a diagram, components which also represent conceptual ideas. Of all the students in this study, only Student A's and Student B's manipulations of their diagrams led them to new interpretations of their initial ideas coupled with new spatial schemes.

For some expert designers, such as Kahn, James Stirling and Daniel Libeskind, the manipulation of conceptual diagrams resulted in changes in both the conceptual domain and the spatial domain. For students, however, establishing the structural correspondence of the conceptual and spatial explorations through conceptual diagrams seemed to be difficult but acquirable with time. Still more difficult for students was understanding that manipulating one of the two domains while coordinating between them might be facilitated through conceptual diagrams; I suggest that understanding this requires a rich mental model. Students may compensate for their lack of a rich mental model by calling upon analogies that are personally meaningful to them.

\section{Conclusion}

Views of creativity often highlight either the importance of incubation in the idea generation process or the significance of sketching as a means of visualizing emergent properties. It would be difficult for students to succeed using either of these processes because of the 
reasons highlighted above. This study investigated the strengths and weaknesses of an alternative approach to design education, one in which students were asked to use conceptual diagrams to formulate and develop a design idea.

The literature on the role of sketching and sketches is rich with evidence of how sketching helps designers realize their imagined ideas or develop new ideas. Furthermore, those who highlight the significance of incubation in creativity suggest that often designers pause when faced with an impasse and take up the problem at a later time with a more productive approach. However, a conception of the design process as one in which ideas emerge suddenly, without much effort, or through purposeless explorations is problematic. Incubation requires intense preliminary work and is not a simple process as described by some designers, and sketching often requires an expert eye to interpret the marks on the paper.

This study suggests that students may manage the design process better in a concept-driven design teaching method that emphasizes the use of conceptual diagrams. The quality of their design work, however, remains related to whether they can construct a meaningful design concept and whether they can convert that concept into a spatial configuration. In contrast to expert architects, whose mental models of design situations include a correlated set of abstract ideas, generic spatial schemes and design examples, students lack rich mental models. For students, analogies may become a useful tool in the construction of a mental model of a design situation.

A concept-driven design teaching method emphasizing the use of conceptual diagrams may help create a classroom environment in which students are focused, committed and motivated. It should be added that students have different learning styles and that different pedagogical approaches would favour different students. With every teaching approach students must be allowed to develop their own understanding. In this particular proposal students first formulated a personally meaningful concept and then used that concept as a yardstick throughout the design process.

During the course the students learned how to look at other examples and abstract them so that they could use them in new design situations. This empowered the students by showing them that they share design ideas with 'master architects' rather than copy them. Students also learned that generic ideas could have different variations and that they could compare and eliminate variations without abandoning their idea.

\section{References}

Alexander, C. (1964). Notes on the Synthesis of Form. London: Pion Press.

Bauer, M. I. and Johnson-Laird, P. N. (1993). 'How diagrams can improve reasoning'. Psychological Science, 4(6): 372-378. 
Boden, M. A. (1991). The Creative Mind: Myths and Mechanisms. New York: Basic Books.

Casakin, H. (2004). 'Visual analogy as a cognitive strategy in the design process: Expert versus novice performance'. Journal of Design Research, 4(2)Casakin, H. P. (2006). 'Assessing the use of metaphors in the design process'. Environment and Planning B, 33(2): 253-268.

Chi, M. T. H., Feltovich, P. and Glaser, R. (1981). 'Categorization and representation of physics problems by experts and novices'. Cognitive Science, 5(2): 121-152.

Clayton, M. J. (2000). 'Diagramming aesthetics: Modernism and architecture in the 21st century'. In Anderson, M., Cheng, P. and Haarslev, V. (eds), Theory and Application of Diagrams: First International Conference, Diagrams 2000, Edinburgh, Scotland, UK, September 2000: Proceedings. New York: Springer, pp. 257-270.

Coyne, R., Snodgrass, A. and Martin, D. (1994). 'Metaphors in the design studio'. Journal of Architectural Education, 48(2): 113-125.

Do, E. Y.-L. and Gross, M. D. (2001). 'Thinking with diagrams in architectural design'. Artificial Intelligence Review, 15(1-2): 135-149.

Dogan, F. (2003). 'The role of conceptual diagrams in the architectural design process: case studies of the First Unitarian Church by Louis Kahn, the Staatsgalerie by Stirling and Wilford Associates, and the Jewish Museum by Daniel Libeskind'. Unpublished PhD Thesis, Atlanta, Georgia Institute of Technology.

Dogan, F. and Nersessian, N. J. (2010). 'Generic abstraction in design creativity: The case of Staatsgalerie by James Stirling'. Design Studies, 31(3): 207-236.

Dogan, F. and Zimring, C. M. (2002). 'Interaction of programming and design: The First Unitarian Congregation of Rochester and Louis I. Kahn'. Journal of Architectural Education, 56(1): 47-56.

Dorst, K. and Cross, N. (2001). 'Creativity in the design process: co-evolution of problem-solution'. Design Studies, 22(5): 425-437.

Ervin, S. M. (1990). 'Designing with diagrams: a role for computing in design education and exploration'. In McCullough, M., Mitchell, W. J. and Purcell, P. (eds), The Electronic Design Studio: Architectural Knowledge and Media in the Computer Era. Cambridge, MA: MIT Press, pp. 108-122.

Gattis, M. and Holyoak, K. J. (1996). 'Mapping conceptual to spatial relations in visual reasoning'. Journal of Experimental Psychology/ Learning, Memory and Cognition, 22(1): 231-240.

Gentner, D. (1983). 'Structure-mapping: A theoretical framework for analogy'. Cognitive Science, 7(2): 155-170.

Gobert, J. D. and Clement, J. J. (1999). 'Effects of studentgenerated diagrams versus student-generated summaries on conceptual understanding of causal and dynamic knowledge in 
plate tectonics'. Journal of Research in Science Teaching, 36(1): 39-53.

Goel, V. and Pirolli, P. (1992). 'The structure of design problem spaces'. Cognitive Science, 16(3): 395-429.

Goldschmidt, G. (1991). 'The dialectics of sketching'. Creativity Research Journal, 4(2): 123-143.

Goldschmidt, G. (1994). 'On visual design thinking: The vis kids of architecture'. Design Studies, 15(2): 158-174.

Goldschmidt, G. (1997). 'Capturing indeterminism: representation in the design problem space'. Design Studies, 18(4): 441-455.

Greeno, J. G. (1989). 'Situations, mental models, and generative knowledge'. In Klahr, D. and Kotovsky, K. (eds), Complex Information Processing: The Impact of Herbert A. Simon. Hillsdale, NJ: Erlbaum, pp. 285-318.

Gross, M. D., Ervin, S. M., Anderson, J. and Fleisher, A. (1987). 'Designing with constraints'. In Kalay, Y. E. (ed.), Computability of Design. New York: Wiley, pp. 53-83.

Hoffmann, D. (1978). Frank Lloyd Wright's Fallingwater: The House and Its History. New York: Dover Publications.

Johnson-Laird, P. N. (1989). 'Analogy and the exercise of creativity'. In Vosniadou, S. and Ortony, A. (eds), Similarity and Analogical Reasoning. Cambridge: Cambridge University Press, pp. 313-331.

Johnson-Laird, P. N. (2002). 'Peirce, logic diagrams, and the elementary operations of reasoning'. Thinking and Reasoning, 8(1): 69-95.

Kahn, L. I. (1961). 'Form and design'. Architectural Design, 31(4): $145-154$.

Lakoff, G. (1987). Women, Fire, and Dangerous Things: What Categories Reveal about the Mind. Chicago, IL: University of Chicago Press.

Lawson, B. (1979). 'Cognitive strategies in architectural design'. Ergonomics, 22(1): 59-68.

Le Corbusier, Eardley, A., Frampton, K., Kolbowski, S. and Institute for Architecture and Urban Studies (1981). Le Corbusier's Firminy Church. New York: Institute for Architecture and Urban Studies: Rizzoli International Publications.

Lloyd, P. and Scott, P. (1994). 'Discovering the design problem'. Design Studies, 15(2): 125-140.

Maher, L. and Tang, H.-H. (2003). 'Co-evolution as a computational and cognitive model of design'. Research in Engineering Design, 14(1): 47-64.

Nersessian, N. J. (2008). Creating Scientific Concepts. Cambridge, MA: MIT Press.

Oxman, R. (1997). 'Design by re-representation: a model of visual reasoning in design'. Design Studies, 18(4): 329-347.

Oxman, R. E. and Oxman, R. M. (1992). 'Refinement and adaptation in design cognition'. Design Studies, 13(2): 117-134. 
Pfeiffer, B. B. and Wright, F. L. (1990). Frank Lloyd Wright Drawings: Masterworks from the Frank Lloyd Wright Archives. New York: Abrams.

Schön, D. (1984). 'The architectural studio as an exemplar of education for reflection-in-action'. Journal of Architectural Education, 38(1): 2-9.

Schön, D. (1987). Educating the Reflective Practitioner: Toward a New Design for Teaching and Learning in the Professions. San Francisco, CA: Jossey-Bass Publishers.

Schön, D. (1988). 'Designing: rules, types and worlds'. Design Studies, 9(3): 181-190.

Schön, D. (1992). 'Designing as reflective conversation with the materials of a design situation'. Research in Engineering Design, 3(3): 131-147.

Schön, D. A. and Wiggins, G. (1992). 'Kinds of seeing and their functions in designing'. Design Studies, 13(2): 135-156.

Shimojima, A. (2001). 'The graphic-linguistic distinction: exploring alternatives'. Artificial Intelligence Review, 15(1-2): 95-114.

Stenning, K. and Lemon, O. (2001). 'Aligning logical and psychological perspectives on diagrammatic reasoning'. Artificial Intelligence Review, 15(1-2): 29-62.

Suwa, M., Gero, J. and Purcell, T. (2000). 'Unexpected discoveries and S-invention of design requirements: Important vehicles for a design process'. Design Studies, 21(6): 539-567.

\section{Biography}

Fehmi Dogan is Assistant Professor of Architecture at the Izmir Institute of Technology. He is interested in design cognition in general and specifically in the use of different representational systems in architectural design. For his doctoral thesis, he conducted archival case studies investigating the role of conceptual diagrams in the design process of Louis I. Kahn, James Stirling and Daniel Libeskind. Currently, he conducts research on design pedagogy and the use of analogy by expert and novice designers. He teaches graduateand undergraduate-level courses on architectural design, research methods, design cognition and representational systems. He has published in journals such as Journal of Architectural Education, Journal of Learning Sciences, Journal of Environmental Psychology and Design Studies. He has presented his work at conferences such as Diagrammatic Reasoning, Cognitive Science Society Conference, and Environmental Design Research Association Conference (EDRA).

\section{Address for Correspondence}

Fehmi Dogan, Faculty of Architecture, Izmir Institute of Technology, Gulbahce, Urla, Izmir 35430, Turkey.

Tel: 902327507050

Fax: 902327507012

Email: fehmidogan@iyte.edu.tr 